\title{
Christine Le Quellec Cottier, Devenir Cendrars. Les années d'apprentissage
}

\section{Emanuele Kanceff}

\section{Q OpenEdition}

1 Journals

\section{Edizione digitale}

URL: http://journals.openedition.org/studifrancesi/35156

DOI: 10.4000/studifrancesi.35156

ISSN: 2421-5856

\section{Editore}

Rosenberg \& Sellier

\section{Edizione cartacea}

Data di pubblicazione: 1 novembre 2005

Paginazione: 448-449

ISSN: 0039-2944

\section{Notizia bibliografica digitale}

Emanuele Kanceff, «Christine Le Quellec Cottier, Devenir Cendrars. Les années d'apprentissage», Studi Francesi [Online], 146 (XLIX | II) | 2005, online dal 30 novembre 2015, consultato il 18 avril 2021. URL: http://journals.openedition.org/studifrancesi/35156; DOI: https://doi.org/10.4000/studifrancesi. 35156

Questo documento è stato generato automaticamente il 18 avril 2021.

\section{(c) (1)}

Studi Francesi è distribuita con Licenza Creative Commons Attribuzione - Non commerciale - Non opere derivate 4.0 Internazionale. 


\title{
Christine Le Quellec Cottier, Devenir Cendrars. Les années d'apprentissage
}

\author{
Emanuele Kanceff
}

\section{NOTIZIA}

CHRISTINE LE QUELLEC COTTIER, Devenir Cendrars. Les années d'apprentissage. Paris, 2004, pp.

328, «Cahiers Blaise Cendrars», 8.

1 Nel primo poema della raccolta Au coeur du monde, del 1918, Blaise Cendrars si presenta senza esitare come «l'uomo che non ha più passato». Questa affermazione perentoria obbliga a guardare davanti a sé, senza voltarsi: dietro non c'è più niente, un terreno deserto. Il poeta ha mantenuto questo principio lungo tutta la sua vita, anche nella sua opera, la sua naissance d'homme è coincisa all'epoca del suo rientro dal fronte, periodo di violenza da cui è tornato alla vita civile mutilato, trasformato.

2 L'autore, rifiutando di seguire il poeta, ha voluto guardare indietro, a costo di rimanere pietrificata. Questo vol arrière è motivato dal desiderio di scoprire l'entrata nel mondo letterario di un ragazzo che poi sarebbe diventato Blaise Cendrars. In effetti, FrédéricLouis Sauser (1887-1961), per le sue origini si trova tra due culture ed egli conosce perfettamente due lingue, è bilingue; in quanto svizzero egli si relaziona sia con la Germania che con la France dell'inizio del Novecento. Nel frattempo, al fine di «s'afficher premier de son nom», Blaise Cendrars cancella la sua formazione tedesca per poter dire di essere stato adottato dalla Francia. Si sceglie uno pseudonimo francese e nell'agosto 1914, firma un manifesto di stranieri pronti ad arruolarsi. Con la copertura del nazionalismo è riuscito a nascondere il suo bagaglio culturale e intellettuale tedesco, a scapito della sua identità. Nello stesso tempo i guizzi della sua memoria hanno permesso l'oblio di un'origine e il risalto dell'altra, liberato da tutti gli obblighi civili e da tutti i legami con la famiglia.

3 Gli studi dei primi scritti del poeta hanno condotto l'autrice a ripassare il quadro cronologico che mi era stato implicitamente dato considerando che la scelta del 
pseudonimo definitivo, nel 1912, segnò il punto di realizzazione di questa sua volontà di ricostruzione di sé stesso. In effetti, Aléas, il romanzo che guida questa ricerca, è conosciuto dai lettori di Blaise Cendrars solo sotto il titolo di Morganni Nameh, pubblicato in numerosi episodi nella rivista parigina «Les Feuilles libres», nel 1922. In più le analisi eseguite a proposito di Aléas, hanno fatto sorgere attraverso dei legami cronologici e tematici in direttai il romanzo di cui ne porta l'impronta nel 1926, Moravagine. Moganni Nameh, nel 1922, di fatto non è che una tappa inaboutie nella direzione dell'universo romanzesco. Secondo una logica molto cendrardiana i testi, gli esseri, i personaggi si sono intrecciati per costruire delle trame molto dense.

Testi e percorsi personali si equivalgono ed è in questa ottica che l'analisi dell'autrice tocca alla fine degli oggetti dimenticati o repudiati dallo scrittore, e degli archivi che hanno valore di testimonianza. Le ricerche condotte in Svizzera, in Francia e in Germania hanno permesso di arricchire e di modificare il ritratto che l'artista ha lasciato di sé. Studiando gli archivi di Berna, l'autore ha potuto analizzare i quaderni della sua giovinezza che sono delle prove preziose che indicano le letture e le preoccupazioni del ragazzo, come anche le traduzioni fatte nel 1910-1911, di testi tedeschi saggi e poemi di autori da lui apprezzati. Nonostante le parole ambigue di Cendrars, manoscritti di questo tipo dimostrano la sua padronanza della lingua tedesca. Le sue collaborazioni a riviste d'avanguardia di Berna e di Monaco, articoli e conferenze confermano d'altronde le sue capacità linguistiche e rafforzano l'impatto di questo universo culturale tedesco nella formazione del poeta, obbligandoci a porci delle domande sul perché di questa negazione. Per di più la messa a disposizione da parte di Miriam Cendrars del quaderno intitolato De Retour, che contiene le note prese durante il ritorno dall'America, permette di osservare sotto una nuova prospettiva la situazione del poeta all'arrivo del suo arrivo in Europa alla fine di giugno $1912 \mathrm{e}$ di scoprire anche come utilizzò o riutilizzò il suo materiale scritto. Lo studio storico ha rivelato l'origine del testo.

$5 \quad$ L'ultima parte di questo lavoro vuole cogliere i risultali testuali di queste ambivalenze centrati sulla produzione e sul ritratto, l'attività e l'isolamento. I tagli sono da leggere come i legami che lo scrittore tesse tra i suoi testi permettendo a certi di esistere a discapito di altri. Quando uscì Moganni Nameh, nel 1922, Moravagine è un romanzo in lavorazione da diversi anni per il quale egli non ha ancora trovato la sua forma definitiva.

6 È stato interessante scoprire dagli archivi conservati che la data 1912 è scritta su una cartella manoscritta, elemento che associa la sparizione di Aléa a quello che non è ancora un personaggio, Moravagine. Questo romanzo famoso è nato prima della guerra e uno studio preciso dell'autore, ha permesso di osservare fino a che punto egli si sia ispirato a Aléa. Aléa conduce a Moravagine ma i due romanzi derivano dallo stesso stampo affettivo: se, come verrà dimostrato Aléa è un tentativo di liberazione dalla colpevolezza per la morte di Elena, la giovane incontrata e amata a San Pietroburgo, la scrittura di Moravagine è, quanto a questo, la necessaria reazione al fallimento di questo primo lavoro: in entrambi i casi, scrivere è un atto di sopravvivenza. 